\title{
Trial designs advance to overcome bitter pill of placebo effect
}

Last month, the Dublin-based drug company Alkermes said it would break from tradition and try a new type of clinical trial design. In an effort to overcome the well-documented placebo effect, it will reassign placebo nonresponders in a second portion of the trial- a notable twist in an industry that some say has not seen enough innovation in trial design in recent decades. The design, known as sequential parallel comparative design, or SPCD, will be used in a phase 3 study for the first time ever of Alkermes's opioid modulator drug ALKS 5461 for major depressive disorder.

If the trial is successful, it could pave the way for other drug companies struggling to overcome the placebo effect, which can be as high as $40 \%$ in trials for disorders that involve self-evaluation of symptoms such as depression, according to Paul Enck, a medical psychologist whose research at the University Hospital Tübingen in Germany focuses on the placebo effect.

Thus far, the primary means of overcoming the placebo effect has been to simply enroll more patients in the trial to attempt to gain enough power to demonstrate the drug is effective or to conduct what is called a placebo run-in, where patients are prescreened for responding or not responding to placebo. But large trials are expensive, and some analyses have found that placebo run-ins, which have been around for decades, are not always effective (Depress. Anxiety 19, 10-19, 2004) and may also prolong drug trials, says Anastasia Ivanova, a biostatistician at the University of North Carolina at Chapel Hill and a consultant for RCT Logic, a Florida-based clinical trial design firm, which licensed the SPCD technique.

By contrast, SPCD is essentially two randomized trials run one after the other, says Maurizio Fava, a psychiatrist at Massachusetts General Hospital (MGH) in Boston, who with his colleagues first proposed the technique in 2003 and holds patents on it (Psychother. Psychosom. 72, 115-127, 2003). In the first stage of the trial, individuals are randomized to receive either a drug or placebo. The difference between SPCD and the standard two-arm randomized trials is that in SPCD, more than half the individuals are assigned to the placebo arm. For example, Alkermes enrolled 142 individuals in its phase 2 trial, 99 of whom received placebo in the first stage. At the end of the first stage, the placebo arm is evaluated and the individuals who did not respond to placebo are re-randomized into another study with half receiving the drug and half receiving the placebo.

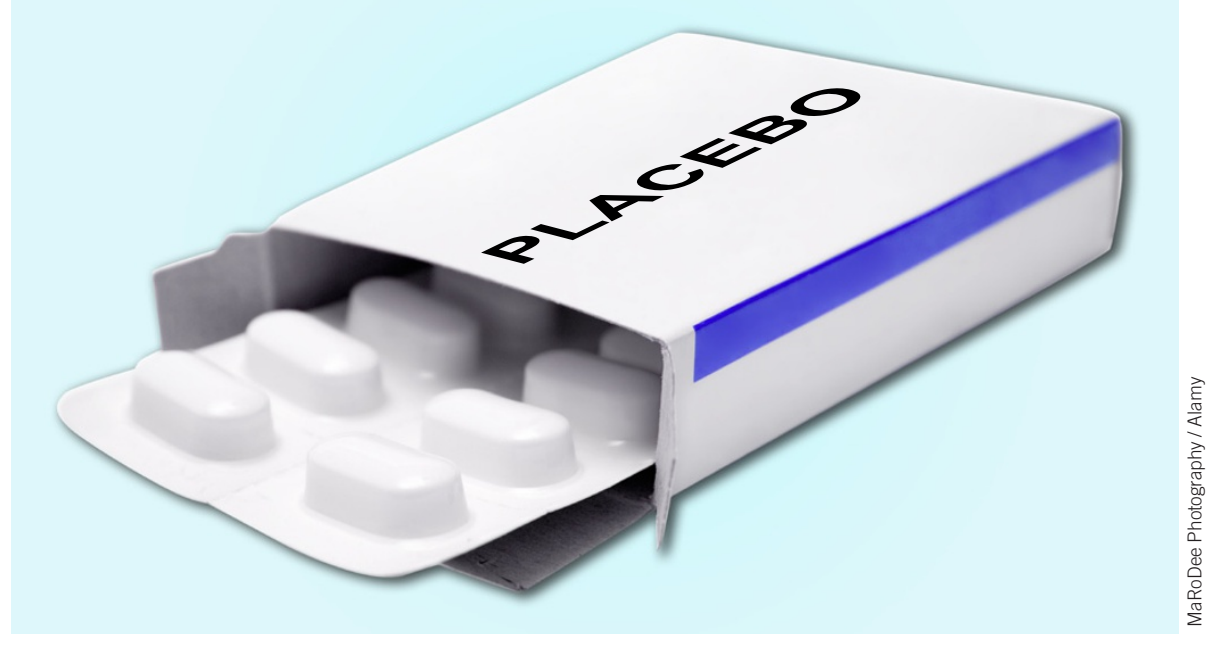

The advantage of such a design is that companies can keep the same level of power and conduct trials with $20 \%$ to $50 \%$ fewer individuals or increase the power of a study by $10 \%$ to $25 \%$, or a combination of both. The design is particularly suited for trials that involve a subjective assessment of drug effect, such as those for pain medications and antidepressants.

Currently, there are 14 completed or ongoing earlier-phase SPCD trials, according to RCT Logic. For example, in a phase 2 SPCD trial of the antipsychotic Abilify (aripiprazole) as an adjunctive treatment to antidepressants, sponsored by New York's Bristol-Myers Squibb (BMS) and conducted by Fava and colleagues at $\mathrm{MGH}$, the placebo response from stage 1 to stage 2 was lowered from $17 \%$ to $8 \%$ (Psychother. Psychosom. 81, 87-97, 2012). This reduction led to an overall $P$ value, a metric used to measure the statistical significance of a study, of 0.17 , compared to what would have been a $P$ value of 0.94 had conventional trial methods been used.

\section{Double duty}

Another trial design, dubbed two-way enrichment design, or TED, is similar to SPCD in that it re-randomizes placebo nonresponders after an initial first stage, but it goes a step further and also re-randomizes drug responders (Stat. Methods Med. Research. doi:10.1177/0962280211431023, 2011). However, pharma has been largely reluctant to incorporate such designs in later-stage drug trials, says Enck. Regulatory agencies "tend to be restrictive of trials that involve any type of unblinding, and companies are not eager to put their drug trials at risk."

Historically, there have been senior members at the FDA who are supportive of innovative trial designs, says Kenneth Kaitin, director of the Tufts Center for the Study of Drug Development in Boston, but the problem is that those individuals are not the same individuals as the FDA reviewers of drug trials, giving drug companies pause. Increasingly, though, "there is more and more buy-in with the agency at the reviewer level that this is a valid and statistically acceptable means," he adds.

Of the larger pharmaceutical companies, BMS is the only company that has thus far used the design in a clinical study, although other companies such as Eli Lilly and Johnson \& Johnson have published articles reviewing the method (Clin. Trials, 4, 309-317, 2007; J. Biopharm. Stat., 4, 737-757, 2012). According to Kaitin, it will probably be the smaller pharmaceutical companies that initially embrace innovative trial designs, and they will work with the FDA earlier on in the drug development process to secure acceptance for the model.

Alkermes's CEO Richard Pops told Nature Medicine that the FDA was very supportive of the SPCD method and that the company plans to use the model for other clinical trials, particularly those for antidepressants. "One great aspect of SPCD is that you can have power with a smaller number of subjects," says Pops. "The general theme of innovative trial design informed by rigorous trial data is the way that Alkermes is thinking about drug development."

Finding new methods of conducting drug trials is imperative, Kaitin says. Companies are "using the same $\mathrm{R} \& \mathrm{D}$ model that was developed 50 years ago," he adds. "The drug development model is so in need of improvement and updating that it's insane."

Monica Heger

Corrected after print 3 December 2013. 


\section{Correction}

In the November 2013 issue, the article entitled "Trial designs advance to overcome bitter pill of placebo effect" (Nat. Med. 19,

1353, 2013) incorrectly quoted Richard Pops as saying that SPCD trials enable power with smaller ends, when it should have said a smaller number of subjects. The error has been corrected in the HTML and PDF

versions of the article. 